\title{
Review
}

\section{Autophagy genes and ageing}

\author{
T Vellai ${ }^{* 1}$
}

Ageing in divergent animal phyla is influenced by several evolutionarily conserved signalling pathways, mitochondrial activity and various environmental factors such as nutrient availability and temperature. Although ageing is a multifactorial process with many mechanisms contributing to the decline, the intracellular accumulation of damaged proteins and mitochondria is a feature common to all aged cells. Autophagy (cellular self-eating) - a lysosome-mediated catabolic process of eukaryotic cells to digest their own constituents - is a major route for the bulk degradation of aberrant cytosolic macromolecules and organelles. Indeed, genetic studies show that autophagy-related genes are required for lifespan extension in various long-lived mutant nematodes and promote survival in worms and flies exposed to prolonged starvation. These data implicate autophagy in ageing control. Furthermore, results in Drosophila demonstrate that promoting basal expression of the autophagy gene Atg8 in the nervous system extends lifespan by $50 \%$, thereby providing evidence that the autophagy pathway regulates the rate at which the tissues age. In this review, the molecular mechanisms by which autophagy genes interact with longevity pathways in diverse organisms ranging from yeast to mammals are discussed.

Cell Death and Differentiation (2009) 16, 94-102; doi:10.1038/cdd.2008.126; published online 12 September 2008

\section{Signalling Pathways and Environmental Factors that Regulate Ageing}

Ageing is an inevitable physiological process characterised by a progressive accumulation of damaged aberrant macromolecules and organelles in somatic cells during the post-developmental period, leading to the decreased ability of the organism to survive. ${ }^{1-4}$ Inefficient removal of nonfunctional aberrant cellular components generated by oxidative damage and a general decline in housekeeping mechanisms appear to be critical in the progression of ageing. ${ }^{5,6}$ Accumulating evidence now shows that changes in the activity of intracellular degradative processes, including the ubiquitin-proteasome system and especially the lysosomemediated autophagic system, are involved in the ageing process and thereby modulate lifespan. Thus, a detailed characterisation of these degradative systems and the elucidation of their regulation might help to understand why and how diverse eukaryotic species age. However, when studying diminishing degradative pathways and their impact on ageing, one might meet two main difficulties. First, because protein degradation is essential for normal growth and development, ${ }^{7-13}$ genetic manipulation interfering with these processes is likely to have pleiotropic effects, for example, it causes premature death, masking any possible specific role in ageing control. Second, uncovering novel genetic determinants of ageing is usually based on the recognition of longlived phenotype caused by reduced activity of pro-ageing genes (Figure 1). The wild-type function of such genes is to accelerate the ageing process, thereby reducing lifespan.
This way, however, antiageing genes that function to slow down the ageing process, that is, the inactivation of which confers accelerated ageing and lifespan shortening, remain largely unexplored. The main cause behind this problem is that most mutations that reduce lifespan often result in serious disorders and kill the animal for reasons independent of ageing. This is particularly obvious in humans; most people do not die due to 'old age', but due to heart disease, stroke, neurodegenerative disorder or cancer. However, the incidence of these pathologies - most of which do not occur in lower eukaryotic models of ageing- correlates with age. In addition, both ageing and age-related diseases are driven by cellular damage; therefore, considerable overlap must exist between the underlying causative pathways.

A remarkable aspect of the ageing process is its highly regulated nature. ${ }^{1,2}$ During the last two decades, a surprisingly large number of genetic factors, chemical substances and environmental cues have been identified that influence ageing in model organisms such as yeast, worms, flies and mice (Figure 1). ${ }^{1-4}$ For example, in the nematode Caenorhabditis elegans, more than 150 pro-ageing genes have been identified the deregulation of which significantly extends lifespan. ${ }^{14,15} \mathrm{~A}$ group of them constitutes the insulin/ IGF-1 (insulin-like growth factor-1) signalling pathway (Figures 1 and 2). Mutant worms with reduced activity of the insulin/IGF-1 receptor DAF-2 live twice as long as the wild type. ${ }^{16}$ Aberrant insulin/IGF-1 receptor activity confers considerable lifespan extension in flies and female mice too. ${ }^{17,18}$ Thus, this conserved hormonal system functions as a longevity pathway in divergent animal species. Indeed, a

\footnotetext{
${ }^{1}$ Department of Genetics, Eötvös Loránd University, Budapest $\mathrm{H}-1117$, Hungary

*Corresponding author: T Vellai, Department of Genetics, Eötvös Loránd University, Budapest, Pázmány Péter stny. 1/C, Budapest H-1117, Hungary.

Tel: + 361209 0555/8684; Fax: + 361209 0555/1841; E-mail: vellai@ falco.elte.hu

Keywords: autophagy; ageing; longevity pathways; cellular toxin; mitochondria; lysosomes

Received 03.6.08; revised 10.7.08; accepted 30.7.08; Edited by G Kroemer; published online 12.9.08
} 


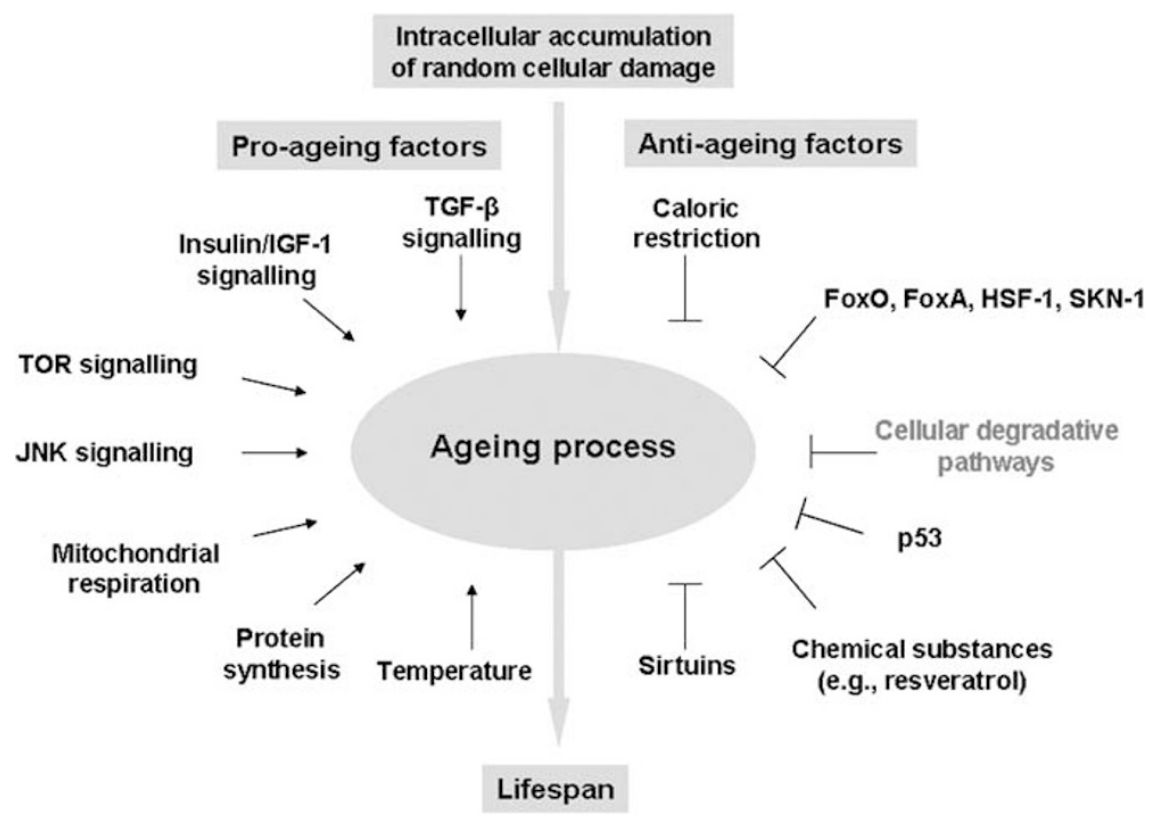

Figure 1 Environmental cues and evolutionarily conserved pathways that regulate the ageing process in diverse eukaryotic phyla. Cellular degradative pathways (highlighted by grey colouring) involve the autophagy pathway and the proteasome system. Arrows indicate activations (pro-ageing factors), bars represent inhibitory interactions (antiageing factors). Ageing, which is multifactorial with many mechanisms contributing to the decline, is driven by the intracellular accumulation of random cellular damage. The overall rate at which damage accumulates is regulated by pathways for maintenance and repair, which in turn may be modulated by metabolic factors

relationship exists between certain genes that are intimately involved in insulin metabolism and the determination of lifespan even in humans. ${ }^{19}$ The insulin/IGF-1 receptor activates a class I phosphatidylinositol-3 kinase (PI3K) that converts phosphatidylinositol $(4,5)$-diphosphate into phosphatidylinositol (3,4,5)-triphosphate (PIP3) (Figure 2). This conversion step is inhibited by the phosphatase and tensin homologue PTEN. PIP3, directly or through the 3phosphoinositide-dependent protein kinase (PDK), further activates a serine-threonine kinase, Akt/PKB (AKT8 virus proto-oncogen/protein kinase $B$ ), which in turn inhibits the activity of the forkhead family transcription factor FoxO. ${ }^{20-24}$ Inactivation of PI3K, PDK and Akt/PKB each extends lifespan, ${ }^{21,22}$ and activated (nuclearly translocated) FoxO is required for lifespan extension in mutants defective in insulin/ IGF-1 signalling. ${ }^{23,24}$ FoxO has been shown to promote longevity in response to reduced insulin/IGF-1 signalling in worms, Drosophila and mammals. ${ }^{23-26}$ Furthermore, results from Drosophila and $C$. elegans demonstrate that the activity of FoxO is also modulated by the c-Jun $\mathrm{N}$-terminal kinase (JNK), a member of the mitogen-activated kinase family. ${ }^{27,28}$ JNK signalling thus acts parallel to insulin/IGF-1 signalling and triggers FoxO to slow down ageing.

In C. elegans, the FoxO-like transcription factor DAF-16 couples the insulin/IGF-1 and transforming growth factor-beta (TGF- $\beta$ ) pathways to control reproductive growth. ${ }^{8}$ Mutants defective in either of the two pathways develop into a non-ageing stress-resistant dauer larval stage (a developmental diapause triggered by crowding and starvation in the wild type) independently of environmental conditions. Inactivation of daf-16 suppresses dauer development in mutant animals with aberrant insulin/IGF-1 or TGF- $\beta$ signalling. Consistent with these findings, TGF- $\beta$ signalling was also found to control longevity in this organism: mutant animals defective in the pathway display up to twofold increases in lifespan. ${ }^{29}$ Regulation of ageing by the TGF- $\beta$ pathway occurs through the translocation of cytosolic DAF-16 into the nucleus. As cross talk between insulin/IGF-1 and TGF- $\beta$ signalling is also evident in mammals, ${ }^{30}$ the TGF- $\beta$ pathway may be involved in the ageing process of higher animal species as well.

Insulin/IGF-1 signalling interacts with the target of rapamycin (TOR) kinase to control metabolism, development and longevity in diverse animal species. ${ }^{31-34}$ TOR, which acts as a sensor of cellular energy levels, is activated by a cascade consisting of Akt/PKB, the tuberous sclerosis complexes 1 and 2 (TSC1 and TSC2) and the Ras homologue enriched in brain (Rheb) (Figure 2). ${ }^{34}$ Signals from transporters that import amino acids into the cytoplasm also activate TOR through the TSC complexes and Rheb. Furthermore, TSC1/2, at least in nematodes, serves as a site where the adenosine monophosphate-activated protein kinase (AMPK) links the cellular AMP/ATP (AMP/adenosine triphosphate) ratio and the insulin/IGF-1-TOR signalling system to lifespan. ${ }^{35}$ The fact that in C. elegans DAF-16/ FoxO directly regulates the transcription of daf-15 that encodes Raptor (regulatory associated protein of TOR) further supports an inherent relationship between insulin/ IGF-1 and TOR signalling. ${ }^{32}$ Accordingly, TOR acts, at least in part, downstream of FoxO in the insulin/IGF-1 signalling pathway to control ageing (Figure 2). Indeed, although strong inhibition of TOR in worms arrests development at the L3 larval stage, decreasing TOR activity starting from the first day of adulthood extends lifespan. ${ }^{31}$ Compromised TOR activity also influences lifespan in yeast, Drosophila and mammals. $^{33,36}$ 


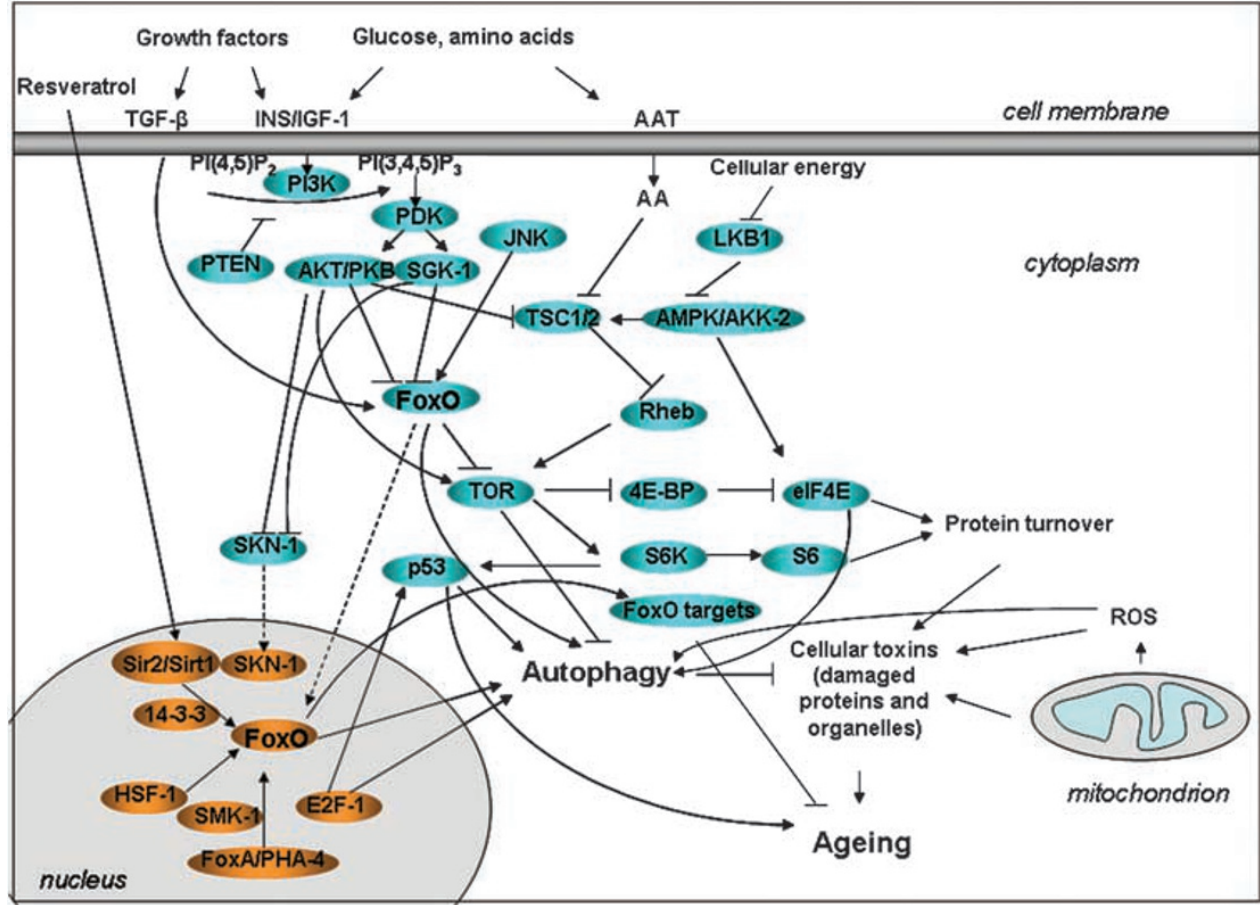

Figure 2 Autophagy in signalling network of ageing. The autophagy pathway interacts with and acts downstream of longevity pathways to regulate diverse cellular functions. AA, amino acid; AAT, amino-acid transporter; AMPK, AMP-activated protein kinase; AKT/PHB, AKT8 virus proto-oncogene/protein kinase B; 4E-BP, eukaryotic initiation factor 4E-binding protein; elF4E, eukaryotic translation initiation factor; E2F-1, transcription factor, a member of the retinoblastoma complex; FoxO, Forkhead transcription factor; HSF-1, heat-shock response transcription factor-1; INS/IGF-1, insulin/insulin-like growth factor-1; LKB1, serine/threonine protein kinase; PDK, 3-phosphoinositide-dependent protein kinase; PHA-4, FoxA transcription factor; PI3K, phosphatidylinositol 3-kinase; PI(4,5) $\mathrm{P}_{2}$, phosphatidylinositol (4,5)-diphosphate; $\mathrm{PI}(3,4,5) \mathrm{P}_{3}$, phosphatidylinositol $(3,4,5)$-triphosphate; PTEN, phosphatase and tensin homologue; Rheb, Ras homologue enriched in brain; ROS, reactive oxygen species; S6K, S6 kinase; S6, small ribosomal subunit S6; Sir2, NAD-dependent histone deacetylase; 14-3-3, small acidic proteins; SKN-1, 'skin in excess' transcription factor-1; SMK-1, a common cofactor of DAF-16/FoxO and PHA-4; TGF- $\beta$, transforming growth factor-beta; TOR, target of rapamycin kinase; TSC1/2, tuberous sclerosis complex-1/2. Solid arrows indicate positive regulatory events; solid bars represent inhibitory interactions; dotted arrows show translocations from the cytoplasm to the nucleus. The autophagy pathway displays multiple signalling cross talks with longevity pathways. Some of the signalling connections identified between these pathways are not shown, due to simplicity of the model. See text for details

Target of rapamycin regulates cell growth by altering mRNA translation in response to nutrient changes. ${ }^{34}$ This kinase promotes protein synthesis through the activation of ribosomal S6 kinase $(\mathrm{S} 6 \mathrm{~K})^{37}$ and inactivation of $4 \mathrm{E}$-binding protein, an inhibitor of eukaryotic translation initiation factor 4E (elF-4E). ${ }^{38}$ elF-4E is a component of a multiprotein complex that directly binds to the 7-methyl-guanosine cap present in almost all mRNAs. If TOR, or more precisely the insulin/IGF-1-TOR signalling axis, promotes both general and specific protein synthesis, one might expect a causative relationship between the regulation of mRNA translation and ageing. Indeed, in Drosophila, the overexpression of dS6K extends lifespan, whereas lowering protein synthesis by inhibition of elF-4E confers increased longevity and oxidative stress resistance in C. elegans. ${ }^{39-42}$

In nematodes, upon stress, DAF-16/FoxO is translocated into the nucleus where it forms a complex with the sirtuin SIR-2.1 to activate the transcription of target genes mediating longevity (Figure 2). ${ }^{43}$ Consistently, increased dosage of SIR-2.1, which alters chromatin structure through its nicotinamide adenine dinucleotide (NAD)-dependent histone deacetylase activity ${ }^{44}$ extends lifespan in C. elegans ${ }^{45}$ Extra copies of sir2 also slow down the ageing process in yeast and Drosophila. ${ }^{46,47}$ Similarly, the mammalian Sirt1 deacetylates
FoxO, and may also be involved in ageing control. Furthermore, sirtuins are activated, at least in mice, by the chemical substance resveratrol having an antiageing role in yeast, worms, flies and mammals. ${ }^{48}$ Molecular association between FoxO and sirtuins requires 14-3-3 proteins, which bind phosphothreonine and phosphoserine residues. ${ }^{43}$ Genetic data indicate that SIR-2.1 and 14-3-3 act in parallel to the insulin/IGF-1 signalling pathway to activate FoxO and lengthen lifespan (Figure 2). Like DAF-16/FoxO, the C. elegans transcription factor heat-shock response transcription factor-1 (HSF-1) involved in heat-shock response is also required for mutations reducing insulin/ IGF-1 signalling to promote longevity. ${ }^{49,50}$ This function of HSF-1 is mediated by its ability to control the transcription of specific target genes it shares with DAF-16/FoxO.

In mammals, the transcription factor p53 has an important function in the detection and elimination of cellular damage by inducing either cellular senescence or apoptosis, and its regulation involves chromatin remodelling factors and insulin/ IGF-1 signalling. ${ }^{51,52}$ In worms and mammals, loss-of-function mutations in the insulin/IGF-1 signalling pathway also antagonise tumour cell growth through activating a p53-mediated cell death pathway. ${ }^{53}$ Together, these results implicate the function of tumour suppressor p53 in ageing 
control. Indeed, enhanced activity of p53, together with its positive regulator Arf, increases cancer resistance and delays ageing in mice. ${ }^{54}$ The linkage of proliferation and longevity to the same signalling pathway strongly supports the co-evolution of tumour resistance and longevity, and may have general relevance to the observation that most animal species become more susceptible to cancer as they age. Thus, p53 is inherently integrated into the signalling network of ageing (Figure 2).

The intracellular accumulation of defective mitochondria occurs progressively during ageing in various animal species. ${ }^{55,56}$ It is now well established that mitochondrial activity is a major source of endogenous reactive oxygen species (ROS) causing oxidative damage of cytosolic materials. ${ }^{57}$ Thus, mitochondria participate in the determination of lifespan (Figure 2). ${ }^{58,59}$ ROS are generally small, short-lived and highly reactive molecules (e.g., oxygen anions, superoxide and hydroxyl radicals, and peroxides) formed by partial reduction of oxygen, which, if they are not detoxified by antioxidising agents, can oxidise macromolecules and damaged organelles. ${ }^{57}$ Oxidation of DNA causes base modifications (mutations) leading to various pathologies in humans, such as cancer, whereas oxidised proteins tend to form aggregates resulting in diverse neurodegenerative pathologies. In addition, accumulation of ROS within the mitochondria may also interfere with the functioning of this organelle due to mitochondrial DNA mutations. Oxidative damage to various constituents of the cell may therefore limit lifespan. Indeed, overexpression of the enzyme superoxide dismutase, which reduces ROS by catabolising superoxides, was shown to extend lifespan in yeast and Drosophila. ${ }^{60,61}$ It must be noted, however, that in another study, antioxidants failed to extend the lifespan of wild-type flies. ${ }^{62}$ In nematodes, lowering the activity of the mitochondrial electron transport chain during development extends adult lifespan. ${ }^{58}$ When mitochondrial respiration is decreased only during adulthood, animals have wild-type lifespan. This indicates that the rate of respiration early in life establishes the rate of ageing in this organism. Furthermore, the longevity produced by mitochondrial dysfunction was reported to be independent of DAF-16/FoxO, and increased much further by reduced insulin/IGF-1 signalling; single mutations in the insulin/IGF-1 pathway or mitochondrial electron transport chain each double the natural lifespan whereas double mutations attenuating both systems confer a lifespan that is extended by up to four times. Thus, mitochondrial respiration functions in parallel to insulin/IGF-1 signalling to influence ageing.

Nutrient availability is a major determinant of lifespan in a variety of organisms. ${ }^{1,4}$ Dietary restriction, prolonged starvation and mutations that cause feeding defects are factors that promote longevity. Temperature is also a growth condition that influences ageing: organisms generally have a faster growth rate and thereby live shorter at higher temperatures. Interestingly, many long-lived mutant animals display increased resistance to nutritional and heat stress. The effects of these environmental cues are mediated, at least
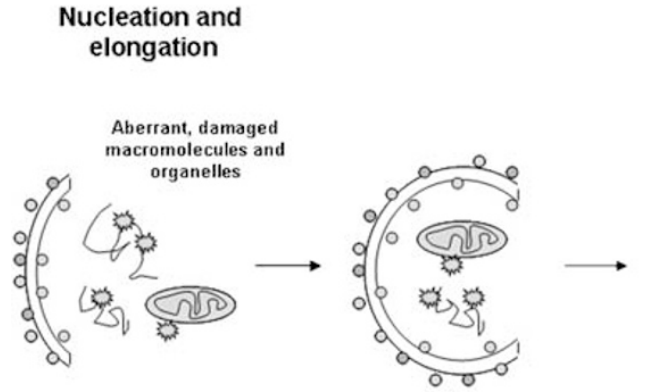

Atg8-PEILC3-II
Atg12-Atg5-Atg16 complex
ROS
Mitochondrion protein

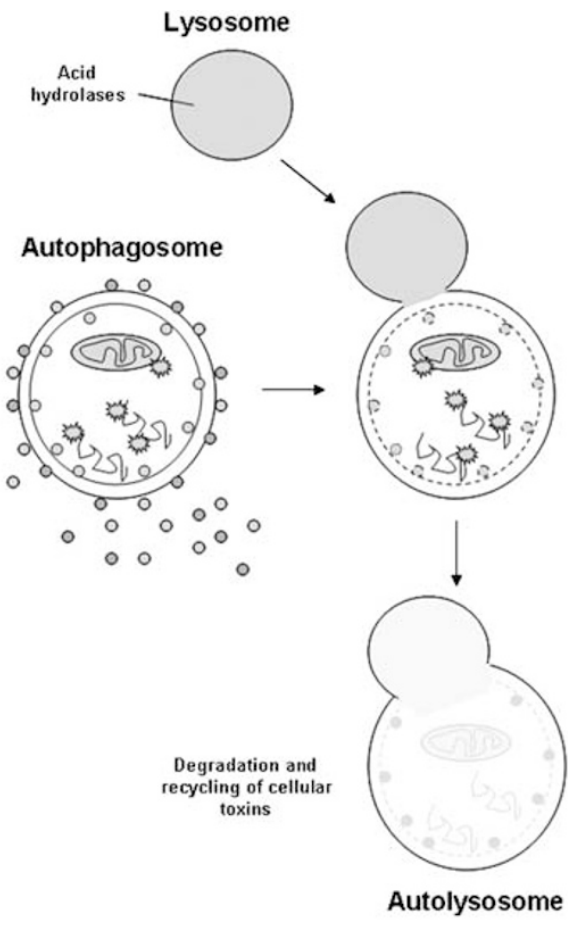

Figure 3 Macroautophagic degradation of oxidative, damaged and aberrant cytosolic materials (cellular toxins) whose intracellular accumulation drives the ageing process. The process of macroautophagy consists of three main stages: initiation of the formation of the isolation membrane/phagophore (nucleation), elongation and completion of the mature autophagosome and fusion of the autophagosome with the lysosome to form an autolysosome. Autophagy proteins including the conjugated Atg8 and the Atg12-Atg5-Atg16 complex are recycled from the outer membrane of the autophagosome. An age-related decline in autophagic activity causes a progressive intracellular accumulation of oxidised, crosslinked proteins and organelles (cellular toxins), leading to a decreased ability of the cell to survive. Autophagy thus regulates cellular homoeostasis. ROS - reactive oxygen species - are generated during respiration in mitochondria and by NADP oxidase and dual oxidase in the cytoplasm 
in part, through the insulin/IGF-1-TOR signalling axis. ${ }^{63}$ This is also true for the oxidative stress response to UV radiation and ROS. ${ }^{64,65}$ In C. elegans, for example, the transcription factor SKN-1 participates in the cellular defence against oxidative stress, ${ }^{66}$ and the activity of this transcription factor was found recently to be under a direct control of certain insulin/IGF-1 signalling kinases, including AKT-1, -2 and SGK-1. ${ }^{65}$ Phosphorylation of $\mathrm{SKN}-1$ by these kinases indicates that the insulin/IGF-1 hormonal system functions to resist oxidative stress in nematodes. Consistently, insulin/ IGF-1 signalling contributes to the increased oxidative stress tolerance in mice too. ${ }^{67}$ Although signalling pathways and environmental factors that influence ageing have been rather well characterised, uncovering which components of cellular metabolism can be fine-tuned to ultimately promote longevity is still an important challenge of ageing research.

\section{Interaction of Longevity Pathways with Autophagy}

Accumulating evidence demonstrates that longevity pathways interact with the autophagic process to regulate diverse cellular functions including growth, differentiation, response to nutrient deprivation and oxidative stress, cell death, as well as macromolecule and organelle turnover. Autophagy is a lysosome-mediated degradative process of eukaryotic cells to digest their own constituents during development or starvation (Figure 3) ${ }^{68-73}$ Depending on the mechanism by which intracellular materials are delivered into the lysosome, there are three general types of autophagy: microautophagy, chaperon-mediated autophagy (CMA) and macroautophagy. In microautophagy, cytoplasmic material is sequestered through direct invagination of the lysosomal membrane. CMA, which is absent in plants, selectively degrades proteins bearing a particular pentapeptide motif (KFERQ) through direct translocation into the lysosome. Macroautophagy involves the formation of subcellular double membrane-bound structures ${ }^{64}$ called autophagosomes to sequester cytoplasmic materials and deliver them into lysosomes for breakdown by acid hydrolases (Figure 3). The products of degradation can be reused for cell functioning. The process of macroautophagy (hereafter referred to as autophagy) starts with the initiation of the formation of the isolation membrane (phagophore), a process called nucleation. The growth of the phagophore (elongation or expansion) terminates in the completion of the autophagosome. Then the fusion of the autophagosome with lysosome forms an autolysosome within which the enclosed material is degraded (Figure 3). ${ }^{71-73}$ The molecular mechanism of autophagy involves several conserved Atg (autophagy-related) proteins, most of which were first identified in yeast. ${ }^{70,72,73}$ Induction of autophagosome formation requires two complexes. These include a lipid signalling complex that contains the class III PI3K Vps34, Atg6/Beclin1, Atg14 and Vps15/p150. ${ }^{73}$ The other complex includes a serine/threonine kinase, Atg1. The kinase activity of Atg1 requires the function of two other autophagy proteins, Atg13 and Atg17. In mammals, which do not contain Atg13, Atg1 was found to associate with the Atg8 orthologues, MAP-LC3 (microtubule-associated protein light chain 3), GATE-16 (Golgi-associated ATPase enhancer of $16 \mathrm{kDa}$ ) and GABARAP ( $\gamma$-aminobutyric acid type A receptor- associated protein). Elongation involves two ubiquitin-like conjugation pathways, the Atg8/MAP-LC3/GABARAP/GATE16 and Atg12 systems. To mediate autophagosome formation, the soluble Atg8 protein undergoes a carboxyl-terminal cleavage by the cysteine protease Atg4 to expose a reactive glycine residue that is activated by the Atg7 (E1-like) and Atg3 (E2-like) enzymes. This function of Atg3 requires a protein complex involving Atg5, Atg12 and Atg16. The activated Atg8 is then covalently linked to phosphatidylethanolamine (Atg8-PE in yeast and lipidated MAP-LC3-II in mammals) and remains bound to the autophagosome membrane until some of it becomes cleaved by Atg 4 to be recycled or if uncleaved gets degraded within the autolysosome. As Atg8 remains covalently bonded to the membrane and may, therefore, be used as a marker for it. Once autophagosome formation is completed, the Atg16-Atg5-Atg12 complex dissociates from its bordering membrane and its components take part in a recycling process mediated by Atg2, Atg9 and Atg18. Then the completed autophagosome is ready for fusion with the endosome or the lysosome (Figure 3).

Autophagy is activated in response to diverse stress and physiological conditions. For example, food deprivation, hyperthermia and hypoxia, which are known as major environmental modulators of ageing, are conditions that also induce autophagy. ${ }^{68,69,73,74}$ At the molecular level, the autophagy pathway displays a remarkable interrelation with factors influencing ageing (Figure 2). For example, in C. elegans, the nutrient-responsive insulin/IGF-1 signalling pathway promotes reproductive growth and prevents dauer development; mutants with reduced insulin/IGF-1 receptor activity develop into dauer larvae independently of growth conditions. Before entering into the dauer stage, the mutant animals were reported to exhibit increased autophagy in cell types considered important for certain dauer-associated morphological changes. ${ }^{75}$ Consistently, disruption of autophagy gene function inhibits dauer morphogenesis and survival. Furthermore, certain autophagy genes have been reported to mediate the effects that insulin/IGF-1 and TGF- $\beta$ signalling have on cell growth. ${ }^{7,8}$ When autophagy gene activity is blocked, nematodes are unable to express a giant body-size phenotype induced by aberrant insulin/IGF-1 or TGF- $\beta$ receptor activity. This indicates that these growth modulatory signalling pathways converge on autophagy genes to control cell size. In mammals, the relationship between autophagy and insulin/IGF-1 signalling appears to be more intimate. Activated FoxO was found recently to increase the transcription of several autophagy-related genes, including Atg8/MAP-LC3, Atg12, Vps34 and Atg6, to induce protein degradation in atrophying muscle cells. ${ }^{76}$ Strikingly, the regulatory effect of FoxO on Atg8 and Atg12 seems to be direct. Starvation or treatment with rapamycin (a specific inhibitor of the nutrient sensor TOR) also rapidly induces an increase in autophagic activity. In yeast, TORC1 (the rapamycin-sensitive Tor kinase complex 1) causes hyperphosphorylation of Atg13, and this form of Atg13 has a lower affinity for Atg1. Inhibition of TORC1 results in partial dephosphorylation of Atg13 and allows its binding to Atg1. ${ }^{77}$ In addition, TORC1 controls phosphorylation of several effectors that regulate transcription or translation of certain proteins, some of which are required for autophagy. It 
cooperatively regulates induction of autophagy with two other partly separate nutrient-sensing pathways that include protein kinase $A$ and Sch9. ${ }^{78}$ In mammals, TOR appears to regulate autophagy in a way similar to that found in yeast. Furthermore, AMPK acting upstream of TOR promotes autophagy in human cell lines and flies. ${ }^{79,80}$ Thus, the insulin/IGF-1-TOR signalling axis controls autophagy at multiple levels (Figure 2).

Normal cell growth requires a well-controlled balance between protein/organelle synthesis and degradation (turnover). Increased growth rate implies higher incidence of the production of aberrant cytosolic components. Irreversibly modified (e.g., oxidised, crosslinked) proteins and defective mitochondria act as cellular toxins, which interfere with normal cellular function. These damages progressively accumulate during ageing, presumably with a higher speed at a higher growth rate, providing a gradually reduced ability of the organism to survive. This might explain why factors that lower mRNA translation to tolerable levels, still enabling essential protein production, increase longevity and cellular resistance to toxic agents. ${ }^{39-42}$ Autophagy functions as a major degradative pathway for removing and recycling damaged non-functional cytoplasmic materials, in particular proteins and organelles. Therefore, regulation of autophagy should be tightly linked to protein synthesis and mitochondrial biogenesis, and includes factors that effectively sense and contribute to the removal of cellular damage. A candidate factor for such work is p53. Indeed, p53, a critical mediator of damage-induced apoptosis, has been shown to induce autophagy in a DRAM (damage-mediated autophagy regulator)-dependent manner to execute a full cell-death response in human cell lines. ${ }^{81,82}$ Furthermore, transcriptional control of p53-mediated (i.e., stress-induced) apoptosis, at least in Drosophila and mammals, involves several chromatin remodelling factors. ${ }^{51}$ For example, E2F-1, which is a component of the mammalian retinoblastoma complex involved in chromatin-mediated transcriptional repression of cell proliferation, regulates apoptosis through activating p53 expression. As p53 functions upstream of the autophagy pathway to induce cell death, ${ }^{72}$ autophagy genes may also be controlled by factors involved in chromatin remodelling. Indeed, recent data show that in humans, E2F-1 binds to the regulatory region and regulates the expression of Atg1, Atg8 and DRAM (Figure 2). ${ }^{83}$

The sirtuin-type chromatin remodelling factors deacetylate FoxO transcription factors thereby promoting longevity in worms, flies and mammals. ${ }^{44-47}$ Recent evidence demonstrates that in mice, Sirt1 is able to induce autophagy in both normal growth and starvation conditions; Sirt1 acetylates Atg5, Atg7 and Atg8 autophagy factors in an $\mathrm{NAD}^{+}$. dependent manner. ${ }^{84}$ It is also intriguing that some aspects of the Sirt1 loss-of-function mutant phenotype highly resemble those of autophagy-defective mice, such as early death and the accumulation of abnormal mitochondria.

Accumulation of ROS, generated mainly during respiration, is an oxidative stress to which cells respond by activating various defence mechanisms. Autophagy has a critical function in the cellular response to oxidative stress (Figure 2). ${ }^{57}$ Several studies show that ROS, as signalling molecules, are able to activate the autophagic process, leading to the subsequent loss of the affected cells. ROS also regulate starvation-induced autophagy.
Moreover, Atg4, an essential protease in the autophagy machinery, has been identified as a direct target for oxidation by ROS. Together, the emerging relationship of autophagy with signalling systems and environmental factors influencing longevity raises the possibility that this facet of cellular metabolism macromolecule and organelle degradation - acts as a determinant of ageing.

\section{Autophagy Genes are Required for Lifespan Extension in Worms and Flies}

The first experimental study implicating autophagy genes in lifespan control was performed by Levine and co-workers. ${ }^{75}$ As the authors showed, RNA interference-mediated depletion of BEC-1 ( $C$. elegans Beclin1 orthologue-1), the worm counterpart of yeast Atg6 and mammalian Beclin1 proteins, inhibits lifespan extension in mutants with reduced DAF-2/ IGF-1 receptor activity. Reduced activity of BEC-1 also shortens adult lifespan in an otherwise wild-type background. However, the life-shortening effects of bec-1 deficiency on daf-2/lgf-1 mutants are more significant than its life-shortening effects on wild-type animals. This suggests that BEC-1 specifically functions in an antiageing process rather than shortening lifespan because its defect is somehow toxic to the animal. BEC-1 is a multifunctional protein. ${ }^{10}$ Therefore, it was important to test whether any other autophagy-related gene similarly affects lifespan. Depletion of ATG-7 and ATG-12 was also found to suppress the long-lived phenotype of daf-2/lgf-1 mutant nematodes. ${ }^{85}$ These results indicate a critical role for the autophagy pathway in promoting longevity. Furthermore, the magnitude by which autophagy genes promote survival is more significant in nematodes and flies that are exposed to prolonged starvation. ${ }^{86,87}$ Thus, autophagy acts as a finetuned cellular pathway to maintain homoeostasis in energy metabolism during period of nutrient deprivation.

bec-1 and atg-7 were also shown to be critical for lifespan extension in eat-2 (eating defective-2) mutant nematodes, in which defects in pharyngeal pumping cause inherent caloric restriction. ${ }^{88-90}$ The longevity response to caloric restriction is known to be mediated by the FoxA-like transcription factor PHA-4 (Figure 2). ${ }^{91}$ PHA-4 is required for increased autophagy in eat-2 mutant worms, suggesting that autophagy is transcriptionally upregulated in response to food limitation. ${ }^{90}$ Furthermore, autophagy genes mediate lifespan extension in mutant nematodes with reduced mitochondrial respiration or reduced TOR activity. ${ }^{89,90}$ These data indicate that the autophagy pathway interacts with and functions downstream of different longevity signals in $C$. elegans (Figure 2). Lipofuscin, which consists of oxidised and crosslinked proteins and lipids, is a pigment that accumulates progressively in ageing tissues. ${ }^{92}$ Mutations in specific autophagy genes cause a more rapid accumulation of lipofuscin during the course of life than it occurs in wild-type animals. ${ }^{78}$

Atg7 mutant Drosophila adults are also short-lived and hypersensitive to nutrient and oxidative stress. ${ }^{87}$ In addition, these mutant flies accumulate ubiquitin-positive protein aggregates in degenerating neurons. Although these results strongly implicate autophagy in ageing control, it cannot be excluded that defects in the process simply eliminate an 
essential pathway for survival and nonspecifically kill the animals.

\section{Autophagy Genes Regulate Ageing in Drosophila}

The potential antiageing function of autophagy could be convincingly demonstrated by the extension of lifespan by enhancing autophagic activity. However, normal cellular functioning and survival requires a balance of catabolic and anabolic pathways. Perturbing the balance in either direction disturbs protein turnover. In the light of this knowledge, it is not surprising that only physiological levels of autophagy can promote survival under stressful conditions (Figure 4) ${ }^{88}$ Conversely, both insufficient and excessive levels of autophagy contribute to premature death. For example, overexpression of certain autophagy-related genes is sufficient to induce high levels of autophagy and results in subsequent loss of the affected cells in Drosophila and mice, ${ }^{9,93}$ and overactivating muscarinic acetylcholine signalling in worms induces excessive autophagy and causes early mortality. ${ }^{86}$ Therefore, there is a need for examining an ageing model with prolonged, but otherwise normally regulated, levels of autophagy. A recent study on Drosophila by Finley and co-workers ${ }^{94}$ provides evidence that under such conditions the autophagy pathway indeed regulates the ageing process. The authors first examined the expression levels of several autophagy genes during adulthood and found a progressive decline in their activity. Remarkably, the age-related loss of autophagy was accompanied by the accumulation of aberrant, ubiquitinated proteins, indicating an essential function for the pathway in the clearance of cytoplasmic debris. These results are highly consistent with those previously obtained from autophagy-deficient mutant mice, which show massive neurodegeneration due to polyubiquitinated protein accumulation. ${ }^{12,13}$ They next assessed the survival of atg 8 loss-of-function mutant files. As discussed above, Atg8 is a conserved ubiquitin-like protein essential for autophagosome formation. Consistent with other data revealing the requirement of autophagy in longevity, Atg8 deficiency shortened adult lifespan, as compared with the wild type. In addition, Atg8 mutant flies also exhibited neurodegenerative phenotypes characterised by the accumulation of insoluble protein aggregates.

As lipid-conjugated Atg8 is essential for nucleation and elongation during autophagosome formation, it has the potential to act as a rate-limiting factor of the autophagic process. Moreover, Atg8 expression is downregulated in neural tissues of older animals as a normal feature of Drosophila ageing ${ }^{94}$ Therefore, it was relevant to ask whether restoring basal levels of Atg8 in the central nervous system late in adulthood influences lifespan in flies. This manipulation was achieved by introducing an Atg8 transgene under the control of an inducible, neuron-specific promoter. Maintaining normal levels of Atg8 mRNA and protein in the nervous system conferred a lifespan that was extended by up to $50 \%$. Importantly, these results have served as genetic evidence that autophagy genes regulate ageing in this organism. Thus, the autophagy pathway appears to be a determinant of the rate at which the tissues age. However, it remains unproven that flies overexpressing Atg8 indeed have more autophagic activity. As most autophagy genes are multifunctional and have pleiotropic effects, these data from Drosophila do not establish that the autophagic process itself is involved in ageing. To address this issue, one should see for example whether longevity induced by maintaining physiological levels of Atg8 late in adulthood can be suppressed by inactivating a gene that has a specific function in autophagy (e.g., Atg18).

In addition to promoting longevity, maintaining physiological levels of Atg8 in the ageing nervous system also prevents the accumulation of protein aggregates. ${ }^{93}$ This suggests that age-dependent decline of autophagy may contribute to the development of several neurodegenerative diseases, which usually become apparent in advanced ages. ${ }^{95,96}$

\section{Cell physiology, pathology}

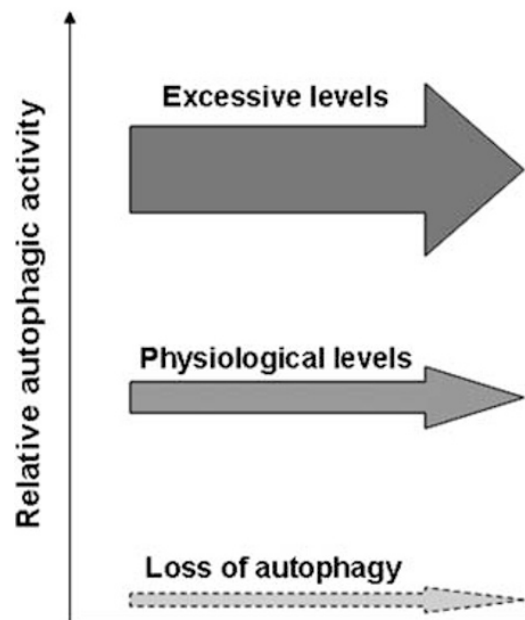

\section{Efficient removal of cellular damage, renewal of cytoplasmic contents, delayed ageing, longevity}

Accumulation of cellular damage (toxins), accelerated ageing

Figure 4 Physiological levels of autophagy are important to maintain cellular homoeostasis, and thereby delay ageing. Both insufficient and excessive levels of autophagy decrease survival. When autophagy is blocked, cellular damage cannot be eliminated. Hyperactivated autophagy mediates autophagic or apoptotic cell death. The activity of autophagy is regulated, at least in part, at the transcriptional level, and adjusted by metabolic factors 


\section{Conclusions and Perspectives}

A progressive intracellular accumulation of oxidative damaged macromolecules and organelles generated by ROS is a hallmark of the ageing process. ${ }^{5,97}$ Although decline in the activity of cellular degradative processes during ageing is well established, it remains unclear whether this phenomenon is merely a consequence of the general deterioration of cellular functions accompanying senescence or an active contributing factor in the ageing process. Results from worms and flies now provide evidence that a causative relationship exists between the regulation of degradative pathways and ageing. Interventions that enhance and prolong protein degradation through promoting physiological levels of autophagy during adulthood enhance longevity. ${ }^{94}$ Conversely, inactivation of cellular degradative pathways, including macroautophagy and other forms of autophagy, that is, CMA and microautophagy, as well as the proteasome system, leads to reduced lifespan as a result of accelerated ageing. ${ }^{98,99}$ Autophagy - the bulk protein degradation pathway - is a major determinant of cellular homoeostasis. It is required for renewal of cytosolic materials, contributing thereby to the rate at which cells accumulate damaged, aberrant proteins and organelles (cellular toxins) (Figure 4).

In nature, different animal species are characterised by markedly different lifespans. For example, mice have relatively short (around 2-year) mean lifespans, whereas humans live for many decades. In these organisms, autophagic activity, which is tightly regulated during development and the reproductive period so that it is maintained at basal levels and induced only when needed, declines during the postreproductive period with a rate specific to a given species. Uncovering the molecular mechanisms underlying the developmental regulation of autophagy will certainly help to understand why and how humans have evolved to a maximum over 70 years. To date, E2F-1, a component of the retinoblastoma complex involved in chromatin-mediated transcriptional regulation, has been identified to promote the activity of Atg1, Atg8 and DRAM in mammals. ${ }^{83}$ Thus, the mechanisms underlying chromatin structure may contribute to the progressive decline in autophagy during ageing.

Acknowledgements. This work was supported by grants from Ministry of Health (ETT 167/2006), Hungarian Scientific Research Funds (OTKA K68372) and the National Office for Research and Technology (RET14/2007 Pázmány Péter program). TV is a grantee of the János Bolyai scholarship.

1. Guarente L, Kenyon C. Genetic pathways that regulate ageing in model organisms. Nature 2000; 408: 255-262

2. Hekimi S, Guarente L. Genetics and the specificity of the aging process. Science 2003 299: 1351-1354.

3. Kirkwood TB, Austad SN. Why do we age. Nature 2000; 408: 233-238.

4. Bishop NA, Guarente L. Genetic links between diet and lifespan: shared mechanisms from yeast to humans. Nat Rev Genet 2007; 8: 835-844.

5. Cuervo AM, Bergamini E, Brunk UT, Dröge W, Ffrench M, Terman A. Autophagy and aging. Importance of maintaining clean cells. Autophagy 2005; 1: 131-140.

6. Rajawat YS, Bossis I. Autophagy in aging and in neurodegenerative disorders. Hormones 2008; 7: 46-61.

7. Aladzsity I, Tóth ML, Sigmond T, Szabó E, Bicsák B, Barna J et al. Autophagy genes unc51 and bec-1 are required for normal cell size in Caenorhabditis elegans. Genetics 2007; 177: 655-660.

8. Vellai T, Bicsák B, Tóth ML, Takács-Vellai K, Kovács AL. Regulation of cell growth by autophagy. Autophagy 2008; 4: 507-509.
9. Scott RC, Gábor J, Neufeld TP. Direct induction of autophagy by Atg1 inhibits cell growth and induces apoptotic cell death. Curr Biol 2007; 17: 1-11.

10. Takács-Vellai K, Vellai T, Puoti A, Passannante M, Wicky C, Streit A et al. Inactivation of the autophagy gene bec-1 triggers apoptotic cell death in C. elegans. Curr Biol 2005; 15: 1513-1517.

11. Fimia GM, Stoykova A, Romagnoli A, Giunta L, Di Bartolomeo S, Nardacci R et al. Ambra1 regulates autophagy and development of the nervous system. Nature 2007; 447: $1121-1125$.

12. Hara T, Nakamura K, Matsui M, Yamamoto A, Nakahara Y, Suzuki-Migishima R et al. Suppression of basal autophagy in neural cells causes neurodegenerative disease in mice. Nature 2006; 441: 885-889.

13. Komatsu M, Waguri S, Chiba T, Murata S, I wata J, Tanida I et al. Loss of autophagy in the central nervous system causes neurodegeneration in mice. Nature 2006; 441: 880-884.

14. Kenyon C. The plasticity of aging: insights from long-lived mutants. Cell 2005; 120: $449-460$.

15. Antebi A. Genetics of aging in Caenorhabditis elegans. PLoS Genet 2007; 3: 1565-1571.

16. Kenyon C, Chang J, Gensch E, Rudner A, Tabtiang R. A C. elegans mutant that lives twice as long as wild type. Nature 1993; 366: 461-464.

17. Tatar M, Kopelman A, Epstein D, Tu MP, Yin CM, Garofalo RS. A mutant Drososphila insulin receptor homolog that extends lifespan and impairs neuroendocrine functions. Science 2001; 292: 107-110.

18. Holzenberger M, Dupont J, Ducos B, Leneuve P, Geloen A, Even PC et al. IGF-1 receptor regulates lifespan and resistance to oxidative stress in mice. Nature 2003; 421: 125-126.

19. Hong MG, Reynolds C, Gatz M, Johansson B, Palmer JC, Gu HF et al. Evidence that the gene encoding insulin degrading enzyme influences human lifespan. Hum Mol Genet 2008; 17: 2370-2378

20. Saltiel AR, Kahn CR. Insulin signalling and the regulation of glucose and lipid metabolism. Nature 2001; 414: 799-806.

21. Morris JZ, Tissenbaum HA, Ruvkun G. A phosphatidylinositol-3-OH kinase family member regulating longevity and diapause in Caenorhaditis elegans. Nature 1996; 382: 536-539.

22. Paradis S, Ailion M, Toker A, Thomas JH, Ruvkun G. A PDK1 homolog is necessary and sufficient to transduce AGE-1 PI3 kinase signals that regulate diapause in Caenorhabditis elegans. Genes Dev 1999; 13: 1438-1452.

23. Lin K, Dorman JB, Rodan A, Kenyon C. daf-16: An HNF-3/forkhead family member that can function to double the life-span of Caenorhabditis elegans. Science 1997; 278: 1319-1321.

24. Ogg S, Paradis S, Gottlieb S, Patterson Gl, Lee L, Tissenbaum HA et al. The fork head transcription factor DAF-16 transduces insulin-like metabolic and longevity signals in C. elegans. Nature 1997; 389: 994-999.

25. Hwangbo DS, Gersham B, Tu M-P, Palmer M, Tatar M. Drosophila dFOXO controls lifespan and regulates insulin signalling in brain and fat body. Nature 2004; 429: 562-566.

26. Salih DAM, Brunet A. FoxO transcription factors in the maintenance of cellular homeostasis during aging. Curr Opin Cell Biol 2008; 20: 126-136.

27. Wang MC, Bohmann D, Jasper H. JNK extends life span and limits growth by antagonizing cellular and organism-wide responses to insulin signaling. Cell 2005; 121: 115-125.

28. Oh SW, Mukhopadhyay A, Svrzikapa N, Jiang F, Davis RJ, Tissenbaum HA. JNK regulates lifespan in Caenorhabditis elegans by modulating nuclear translocation of forkhead transcription factor DAF-16. Proc Natl Acad Sci USA 2005; 102: 4494-4499.

29. Shaw WM, Luo S, Landis J, Ashraf J, Murphy CT. The $C$. elegans TGF- $\beta$ dauer pathway regulates longevity via insulin signalling. Curr Biol 2007; 17: 1635-1645.

30. Danielpour D, Song K. Cross-talk between IGF-I and TGF-b signaling pathways. Cytokine Growth Factor Rev 2006; 17: 59-74.

31. Vellai T, Takács-Vellai K, Zhang Y, Kovács AL, Orosz L, Müller F. Influence of TOR kinase on lifespan in C. elegans. Nature 2003; 426: 620.

32. Jia K, Chen $\mathrm{D}$, Riddle $\mathrm{DL}$. The TOR pathway interacts with the insulin signaling pathway to regulate C. elegans larval development, metabolism and life span. Development 2004; 131: 3897-3906.

33. Kapahi P, Zid BM, Harper T, Koslover D, Sapin V, Benzer S. Regulation of lifespan in Drosophila by modulation of genes in the TOR signaling pathway. Curr Biol 2004; 14: 885-890.

34. Oldham S, Hafen E. Insulin/IGF and target of rapamycin signaling: a TOR de force in growth control. Trends Cell Biol 2003; 13: 79-85.

35. Apfeld J, O'Connor G, McDonagh T, DiStefano PS, Curtis R. The AMP-activated protein kinase AAK-2 links energy levels and insulin-like signals to lifespan in C. elegans. Genes Dev 2004; 18: 3004-3009.

36. Kaeberlein M, Powers III WR, Steffen KK, Westman EA, Hu D, Dang N et al. Regulation of yeast replicative life span by TOR and Sch9 in response to nutrients. Science 2005; 310: 1193-1196.

37. Dufner A, Thomas G. Ribosomal S6 kinase signaling and the control of translation. Exp Cell Res 1999; 253: 100-109.

38. Sonenberg N, Gingras AC. The mRNA 50 cap-binding protein elF4E and control of cell growth. Curr Opin Cell Biol 1998; 10: 268-275.

39. Syntichaki P, Troulinaki K, Tavernarakis N. elF4E function in somatic cells modulates ageing in Caenorhabditis elegans. Nature 2007; 445: 922-926.

40. Hansen M, Taubert S, Crawford D, Libina N, Lee S-J, Kenyon C. Lifespan extension by conditions that inhibit translation in Caenorhabditis elegans. Aging Cell 2007; 6 : 95-110. 
41. Pan KZ, Palter JE, Rogers AN, Olsen A, Chen D, Lithgow GJ et al. Inhibition of mRNA translation extends lifespan in Caenorhabditis elegans. Aging Cell 2007; 6: 111-119.

42. Tavernarakis N. Ageing and the regulation of protein synthesis: a balancing act? Trends Cell Biol 2008; 18: 228-235.

43. Berdichevsky A, Viswanathan M, Horvitz RH, Guarente L. C. elegans SIR-2.1 interacts with 14-3-3 proteins to activate DAF-16 and extend life span. Cell 2006; 125: 1165-1177.

44. Imai S, Armstrong CM, Kaeberlein M, Guarente L. Transcriptional silencing and longevity protein Sir2 is an NAD-dependent histone deacetylase. Nature 2000; 403: 795-800.

45. Tissenbaum HA, Guarente L. Increased dosage of a sir-2 gene extends lifespan in Caenorhabditis elegans. Nature 2001; 410: 227-230.

46. Kaeberlein M, McVey M, Guarente L. The SIR2/3/4 complex and SIR2 alone promote longevity in Saccharomyces cerevisiae by two different mechanisms. Genes Dev 1999; 13 2570-2580.

47. Wood JG, Rogina B, Lavu S, Howitz K, Helfand SF, Tatar M et al. Sirtuin activators mimic caloric restriction and delay ageing metazoans. Nature 2004; 430: 686-689.

48. Baur JA, Pearson KJ, Price NL, Jamieson HA, Lerin C, Kalra A et al. Resveratrol improves health and survival of mice on a high-calorie diet. Nature 2006; 444: 337-341.

49. Hsu A-L, Murphy CT, Kenyon C. Regulation of aging and age-related disease by DAF-16 and heat-shock factor. Science 2003; 300: 1142-1145.

50. Morley JF, Morimoto RI. Regulation of longevity in Caenorhabditis elegans by heat shock factor and molecular chaperones. Mol Biol Cell 2004; 15: 657-664.

51. Kumar S, Cakouros D. Transcriptional control of the core cell-death machinery. Trends Biochem Sci 2004; 29: 193-199.

52. Maier B, Gluba W, Bernier B, Turner T, Mohammad K, Guise T et al. Modulation of mammalian life span by the short isoform of p53. Genes Dev 2004; 18: 306-319.

53. Pinkston JM, Garigan D, Hansen M, Kenyon C. Mutations that increase the life span of C. elegans inhibit tumor growth. Science 2006; 313: 971-975.

54. Matheu A, Maraver A, Klatt P, Flores I, Garcia-Cao I, Borras C et al. Delayed ageing through damage protection by the Arf/p53 pathway. Nature 2007; 448: 375-380.

55. Terman A. Catabolic insufficiency and aging. Ann NY Acad Sci 2006; 1067: 27-36.

56. Bua E, Johnson J, Herbst A, Delong B, McKenzie D, Salamat S et al. Mitochondrial DNAdeletion mutations accumulate intracellularly to detrimental levels in aged human skeletal muscle fibers. Am J Hum Genet 2006; 79: 469-480.

57. Scherz-Shouval R, Elazar Z. ROS, mitochondria and the regulation of autophagy. Trends Cell Biol 2007; 17: 422-427.

58. Dillin A, Hsu AL, Arantes-Oliveira N, Lehrer-Graiwer J, Hsin H, Fraser AG et al. Rates of behavior and aging specified by mitochondrial function during development. Science 2002 298: 2398-2401.

59. Lee SS, Lee RY, Fraser AG, Kamath RS, Ahringer J, Ruvkun G. A systematic RNAi screen identifies a critical role for mitochondria in C. elegans longevity. Nat Genet 2003; 33: 40-48.

60. Parkes TL, Elia AJ, Dickinson D, Hilliker AJ, Phillips JP, Boulianne GL. Extension of Drosophila lifespan by overexpression of human SOD1 in motorneurons. Nat Genet 1998; 19: 171-174.

61. Longo VD, Liou LL, Valentine JS, Gralla EB. Mitochondrial superoxide decreases yeast survival in stationary phase. Arch Biochem Biophys 1999; 365: 131-142.

62. Magwere T, West M, Riyahi K, Murphy MP, Smith RA, Partridge L. The effects of exogenous antioxidants on lifespan and oxidative stress resistance in Drosophila melanogaster. Mech Aging Dev 2006; 127: 356-370.

63. Walker G, Houthoofd K, Vanfleteren JR, Gems D. Dietary restriction in C. elegans from rate-of-living effects to nutrient sensing pathways. Mech Aging Dev 2005; 126: 929-937.

64. Franceschia C, Olivieria F, Marchegiania F, Cardellia M, Cavallonea L, Caprib M et al. Genes involved in immune response/inflammation, IGF1/insulin pathway and response to oxidative stress play a major role in the genetics of human longevity: the lesson of centenarians. Mech Ageing Dev 2005; 126: 351-361.

65. Tullet JMA, Hertweck M, An JH, Baker J, Hwang Y, Liu S et al. Direct inhibition of the longevity-promoting factor SKN-1 by insulin-like signaling in C. elegans. Cell 2008; 132: 1025-1038.

66. An JH, Blackwell TK. SKN-1 links $C$. elegans mesendodermal specification to a conserved oxidative stress response. Genes Dev 2003; 17: 1882-1893.

67. Beyer TA, Xu W, Teupser D, auf dem Keller U, Bugnon P, Hildt E et al. Impaired liver regeneration in Nrf2 knockout mice: role of ROS-mediated insulin/IGF-1 resistance. EMBO J 2008; 27: 212-223.

68. Klionsky DJ, Emr SD. Autophagy as a regulated pathway of cellular degradation. Science 2000; 290: 1717-1721.

69. Cuervo AM. Autophagy: in sickness and in health. Trends Cell Biol 2004; 14: 70-77.

70. Klionsky DJ. The molecular machinery of autophagy: unanswered questions. J Cell Sci 2005; 118: 7-18

71. Kovács AL, Pálfia Z, Réz G, Vellai T, Kovács J. Sequestration revisited. Integrating traditional electron microscopy, de novo assembly and new results. Autophagy 2007; 3: 655-662.
72. Suzuki K, Ohsumi Y. Molecular machinery of autophagosome formation in yeast, Saccharomyces cerevisiae. FEBS Lett 2007; 581: 2156-2161.

73. Levine B, Kroemer G. Autophagy in the pathogenesis of disease. Cell 2008; 132 27-42.

74. Chu CT. Eaten alive: autophagy and neuronal cell death after hypoxia-ischemia. $A m \mathrm{~J}$ Pathol 2008; 172: 284-287.

75. Meléndez A, Tallóczy Z, Seaman M, Eskelinen E-L, Hall DH, Levine B. Autophagy genes are essential for dauer development and life span extension in C. elegans. Science 2003 301: 1387-1391.

76. Zhao J, Brault JJ, Schild A, Cao P, Sandri M, Schiaffino S et al. FoxO3 co-ordinately activates protein degradation by the autophagic/lysosomal and proteasomal pathways in atrophying muscle cells. Cell Metab 2007; 6: 472-483.

77. Noda T, Ohsumi Y. Tor, a phosphatidylinositol kinase homologue, controls autophagy in yeast. J Biol Chem 1998; 273: 3963-3966.

78. Yorimitsu T, Zaman Z, Broach JR, Klionsky DJ. Protein kinase A and Sch9 coopersatively regulate induction of autophagy in Saccharomyces cerevisiae. Mol Biol Cell 2007; 18 4180-4189.

79. Liang J, Shao SH, Xu ZX, Hennessy B, Ding Z, Larrea M et al. The energy-sensing LKB1AMPK pathway regulates p27(kip1) phosphorylation mediating the decision to enter autophagy or apoptosis. Nat Cell Biol 2007; 9: 218-224.

80. Lippai M, Csikós G, Maróy P, Lukácsovich T, Juhász G, Sass M. SNF4A AMPK $\gamma$ subunit is required for regulation of developmental and stress-induced autophagy. Autophagy 2008; 4: 476-486.

81. Crighton D, Wilkinson S, O'Prey J, Syed N, Smith P, Harrison PR et al. DRAM, p53-induced modulator of autophagy, is critical for apoptosis. Cell 2006; 126: 121-134.

82. Tasdemir E, Maiuri MC, Galluzzi L, Vitale I, Djavaheri-Mergny M, D'Amelio M et al. Regulation of autophagy by cytoplasmic p53. Nat Cell Biol 2008; 10: 676-687.

83. Polager S, Ofir M, Ginsberg D. E2F1 regulates autophagy and the transcription of autophagy genes. Oncogene 2008; 27: 4860-4864.

84. Lee IH, Cao L, Mostoslavsky R, Lombard DB, Liu J, Bruns NE et al. A role for the NAD-dependent deacetylase Sirt1 in the regulation of autophagy. Proc Natl Acad Sci USA 2008; 105: 3374-3379.

85. Hars ES, Qi H, Ryazanov AG, Jin S, Cai L, Hu C et al. Autophagy regulates aging in C. elegans. Autophagy 2007; 3: 93-95.

86. Kang C, You Y, Avery L. Dual roles of autophagy in the survival of Caenorhabditis elegans during starvation. Genes Dev 2007; 21: 2161-2171.

87. Juhász G, Erdi B, Sass M, Neufeld TP. Atg7-dependent autophagy promotes neurona health, stress tolerance and longevity but is dispensable for metamorphosis in Drosophila. Genes Dev 2007; 21: 3061-3066.

88. Jia K, Levine B. Autophagy is required for dietary restriction-mediated life span extension in C. elegans. Autophagy 2007; 3: 597-599

89. Tóth ML, Sigmond T, Borsos É, Barna J, Erdélyi P, Takács-Vellai $\mathrm{K}$ et al. Longevity pathways converge on autophagy genes to regulate life span in Caenorhabditis elegans. Autophagy 2008; 4: 330-338.

90. Hansen M, Chandra A, Mitic LL, Onken B, Driscoll M, Kenyon C. A role for autophagy in the extension of lifespan by dietary restriction in C. elegans. PLOS Genet 2008; 4: e24

91. Panowski SH, Wolff S, Aguilaniu H, Durieux J, Dillin A. PHA-4/Foxa mediates diet-restriction-induced longevity of $C$. elegans. Nature 2007; 447: 550-555.

92. Herndon LA, Schmeissner PJ, Dudaronek JM, Brown PA, Listner KM, Sakano Y et al. Stochastic and genetic factors influence tissue-specific decline in ageing $C$. elegans. Nature 2002; 419: 808-814.

93. Erlich S, Shohami E, Pinkas-Kramarski R. Neurodegeneration induces upregulation of Beclin 1. Autophagy 2006; 2: 49-51.

94. Simonsen A, Cumming RC, Brech A, Isakson P, Schubert DR, Finley KD. Promoting basal levels of autophagy in the nervous system enhances longevity and oxidative stress in adult Drosophila. Autophagy 2008; 4: 176-184.

95. Rubinsztein DC, DiFiglia M, Heintz N, Nixon RA, Qin Z-H, Ravikumar B et al. Autophagy and its possible roles in nervous system diseases, damage and repair. Autophagy 2005; 1 $11-22$.

96. Rubinsztein DC. The roles of intracellular protein-degradation pathways in neurodegeneration. Nature 2006; 443: 780-786.

97. Hekimi S, Guarente L. Genetics and the specificity of the aging process. Science 2003; 299: 1351-1354.

98. Ghazi A, Henis-Korenblit S, Kenyon C. Regulation of Caenorhabditis elegans life span by a proteasomal E3 ligase complex. Proc Natl Acad Sci USA 2007; 104: 5947-5952.

99. Mizushima N, Levine B, Cuervo AM, Klionsky DJ. Autophagy fights disease through cellular self-digestion. Nature 2008; 451: 1069-1074. 